\title{
Isolated Plantar Vein Thrombosis Resembling a Corn with a Bruise
}

\author{
Ji Eun Hahm, Kang Su Kim, Jae Won Ha, Chul Woo Kim, Sang Seok Kim \\ Department of Dermatology, Kangdong Sacred Heart Hospital, College of Medicine, Hallym University, Seoul, Korea
}

Plantar vein thrombosis, rarely-reported disease, is usually accompanied by pain and tenderness in the plantar region and should be differentiated from other dermatological conditions causing plantar pain, such as hemorrhagic corn/callus, plantar epidermal cyst, verruca, or plantar fibromatosis. A 52-year-old man presented with a violaceous tender subcutaneous nodule overlying a hyperkeratotic plaque on his sole. Initially, he thought it was a corn and applied keratolytic agents, which failed to work. Sonography revealed a well-demarcated mass with increased peripheral vascularity. His pain was relieved after a complete wide excision, which confirmed the mass to be plantar vein thrombosis after histopathological examination. (Ann Dermatol 31(1) 66 69, 2019)

\section{-Keywords-}

Corn, Plantar vein thrombosis, Venous thrombosis

\section{INTRODUCTION}

Plantar pain caused by dermatological condition is often associated with plantar epidermal cyst, hemorrhagic corn

Received May 2, 2017, Revised November 27, 2017, Accepted for publication December 31, 2017

Corresponding author: Chul Woo Kim, Department of Dermatology, Kangdong Sacred Heart Hospital, College of Medicine, Hallym University, 150 Seongan-ro, Gangdong-gu, Seoul 05355, Korea. Tel: 82-2-2224-2285, Fax: 82-2-474-7913, E-mail: hje150273@naver.com ORCID: https://orcid.org/0000-0002-0970-0439

This is an Open Access article distributed under the terms of the Creative Commons Attribution Non-Commercial License (http://creativecommons. org/licenses/by-nc/4.0) which permits unrestricted non-commercial use, distribution, and reproduction in any medium, provided the original work is properly cited.

Copyright (c) The Korean Dermatological Association and The Korean Society for Investigative Dermatology or callus, plantar fibromatosis, or plantar verruca ${ }^{1}$. Among laborers, they may develop from excess pressure on the bony prominences of the feet, repetitive uneven friction from footwear, or gait abnormalities. Plantar vein thrombosis is a rare condition causing plantar pain. The exact cause of plantar vein thrombosis is yet unclear, but predisposing conditions, such as prior trauma, surgery, paraneoplastic syndromes, or coagulation disorders have been described. To date, there is no established treatment except surgical excision, but reportedly, nonsteroidal anti-inflammatory drug or heparin with elastic bandage is known to be effective for symptomatic control ${ }^{2-5}$. To the best of our knowledge, this is the typical case of an isolated plantar vein thrombosis resembling a corn or verruca with a bruise, which showed improvement after surgical excision, in a man working as a freight car driver. We received the patient's consent form about publishing all photographic materials.

\section{CASE REPORT}

A 52-year-old man working as a freight car driver presented to our clinic with a tender nodule on his right sole toward the heel, which developed 4 years ago and got bigger. He thought it was a corn and applied some keratolytic agents, with which it failed to improve. Physical examination revealed a walnut-sized, firm, fixed subcutaneous nodule with an overlying fine violaceous hyperkeratotic plaque on the heel side of the right plantar area (Fig. 1A). Clinically, he was diagnosed as having a corn or verruca associated with a neighboring bruise. Sonography was performed to rule out the presence of an underlying subcutaneous tumor, and revealed a $1.9 \times 1.2 \mathrm{~cm}$ sized, well-demarcated, lobulated heterogeneous hypoechoic mass surrounded by a thin anechoic area with increased peripheral vascularity (Fig. 2). The radiologist suggested an 

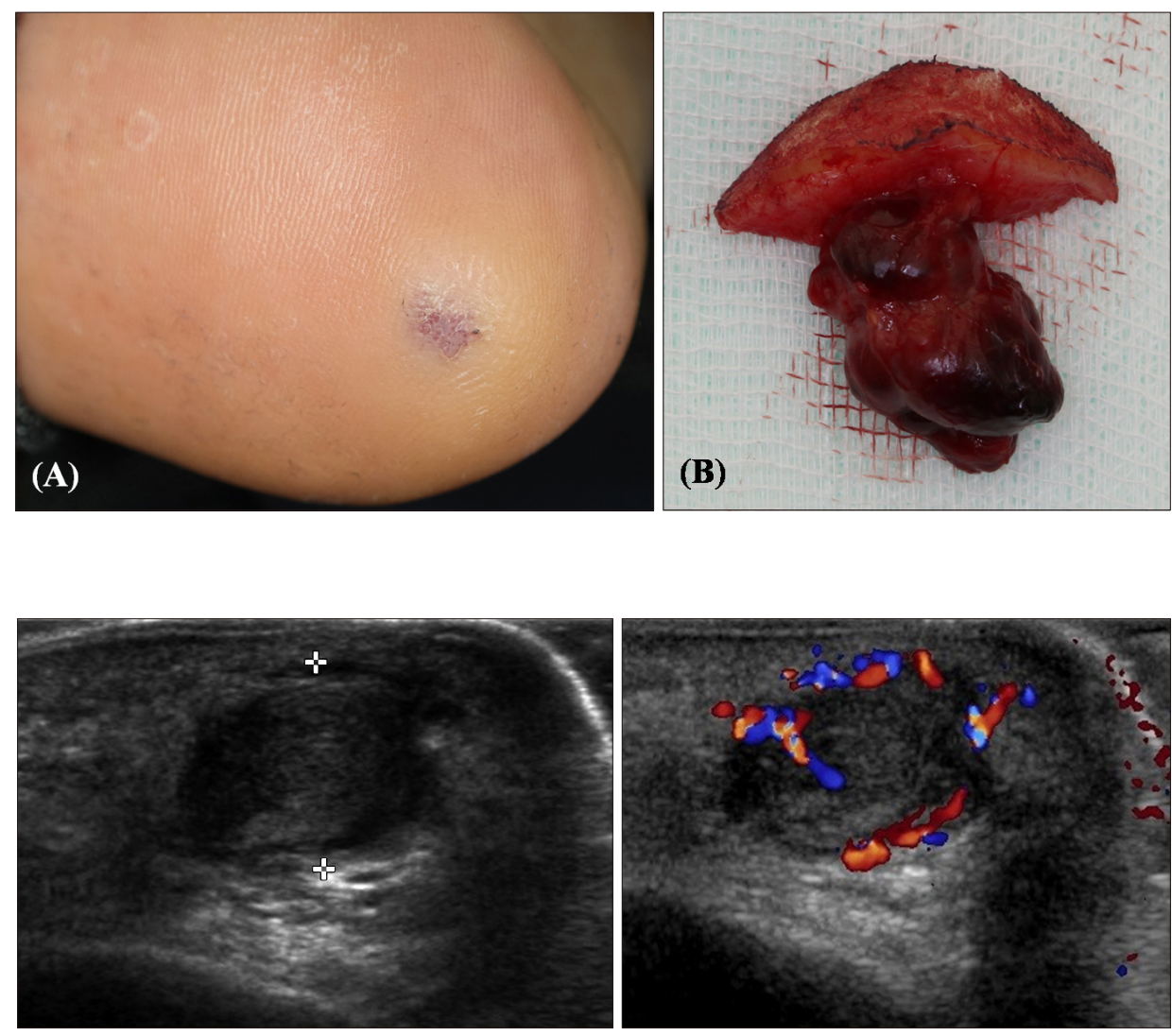

Fig. 2. A $1.9 \times 1.2 \mathrm{~cm}$ sized, wellmarginated, lobulated, heterogeneous hypoechoic mass surrounded by a thin anechoic area with increased peripheral vascularity.
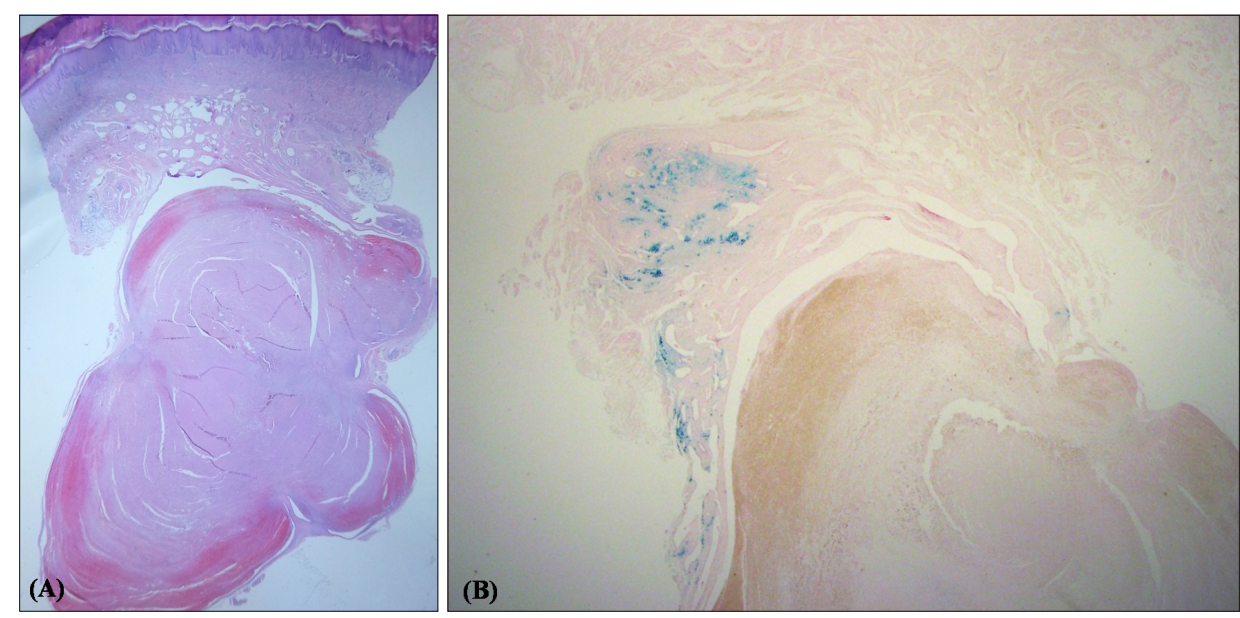

Fig. 3. (A) Several variable thick and plicated walled small muscular vessels in the dermis and its abutting thrombus consisting of aggregated erythrocytes and eosinophilic fibrin in the lumen of a dilated vein in the subcutaneous layer $(\mathrm{H} \& \mathrm{E}, \times 10)$. (B) Granular bluish deposit (hemosiderin) within and around mural walls of muscular vessels (Iron stain, $\times 20$ ).

inflamed epidermal cyst. Surgical excision was performed for complete removal of the superficial corn and the subcutaneous tumor, to relieve his plantar tenderness. A dark erythematous rubbery nodule, $2 \mathrm{~cm}$ in diameter, was found in the subcutaneous layer (Fig. 1B). There was minimal intraoperative bleeding. Histopathological examination revealed several variable thick and plicated walled small muscular vessels and hemosiderin deposition within and around them in focal areas of the dermis. The subcutaneous layer showed a large vessel whose lumen was blocked by a thrombus consisting of aggregated erythrocytes and eosinophilic fibrin (Fig. 3A). The violaceous bruise-like macule around the nodule was turned out dermal deposits of hemosiderin (Fig. 3B). Based on these findings, he was diagnosed as having an isolated plantar vein thrombosis. Sutures were removed 2 weeks later, and he had no recurrence during 6-month follow up. 


\section{DISCUSSION}

Plantar vein thrombosis was first reported in 1997 by Legrand et al. ${ }^{6}$ in a patient developing this condition after a saphenectomy. The exact etiology is yet unknown, but reportedly, it has been associated with trauma or athletic activity $^{2}$, postsurgical immobilization ${ }^{5,6}$, coagulation disorders such as anticardiolipin syndrome ${ }^{4}$ or prothrombin G20210A mutation ${ }^{3}$ and paraneoplastic syndromes in a patient with bone metastasis ${ }^{2}$. Our case was not associated with prior surgery, malignancy, or coagulation disorders and might have been caused by repetitive trauma because as a freight car driver, he was known to have carried heavy loads.

Long-standing subcutaneous venous thrombosis might cause both tender subcutaneous mass and surface hyperkeratosis of the epidermis, which makes diagnosis more difficult. It should be differentiated from other dermatological conditions causing plantar pain such as hemorrhagic corn or callus, plantar epidermal cyst, plantar fibromatosis, and plantar verruca ${ }^{1}$. A corn or callus is the localized focus of hyperkeratosis, arising on the plantar portion from foot deformity or pressure, and the accompanying pain is usually relieved by paring it or applying keratolytic agent ${ }^{7}$. Plantar warts appear as horny plugs surrounded by a ring of hyperkeratotic skin with slight elevation $^{7}$. They also revealed homogeneous black to red dots and globules with papilliform surfaces more prominently seen via dermoscopy ${ }^{8}$. In the case of plantar epidermoid cyst, there is a tiny blackhead plugging the central punctum of the cyst and sometimes foul smelling cheesy debris drains from it ${ }^{9}$. Typically, plantar fibromatosis presents along the central or medial aspect of the plantar fascia as a firm, fixed, rather deep subcutaneous nodule ${ }^{10}$.

To make a proper diagnosis, radiological studies of the lesion may be essential. Plantar vein thrombosis can be diagnosed with Doppler sonography or magnetic resonance imaging (MRI). On Doppler sonography, it characteristically appears as hypoechoic, dilated, noncompressible vessels with no luminal blood flow ${ }^{2}$. However, being operator dependent, a diagnosis might be missed, and MRI is preferred for evaluating persistent foot pain ${ }^{11}$. The typical MRI finding is intraluminal filling defect in plantar veins in association with perivenular enhancement ${ }^{12}$. On the other hand, plantar fibromatosis shows ill-defined, infiltrative mass occurring in the deep aponeurosis adjacent to the plantar muscles with heterogeneous signal intensity equal to or less than that of skeletal muscle on both T1- and T2-weighted MRI ${ }^{10}$. Unruptured epidermal inclusion cysts have intermediated to slightly increased signal intensity on
T1-weighted images and high signal intensity on T2weighted/fluid-sensitive sequences ${ }^{13}$. Finally, biopsy, preferentially excisional one, can help confirm the diagnosis. We removed the thrombosed vein surgically with minimal intraoperative bleeding and the patient had a 6-month follow up without recurrence. Therefore, surgical excision should be considered as a treatment option for plantar vein thrombosis.

In conclusion, plantar vein thrombosis is one of the rare dermatologic diseases causing plantar pain. This is the case that looked like a corn associated with a bruise, perhaps due to its long-standing nature and hemosiderin deposition. When dermatologists encounter patients with painful plantar subcutaneous mass, multiple disorders should be considered in the differential diagnosis. Radiological studies or skin biopsy can be a useful tool for the diagnosis.

\section{CONFLICTS OF INTEREST}

The authors have nothing to disclose.

\section{ORCID}

Ji Eun Hahm, https://orcid.org/0000-0001-7863-2452

Kang Su Kim, https://orcid.org/0000-0002-2996-9814

Jae Won Ha, https://orcid.org/0000-0003-2097-7241

Chul Woo Kim, https://orcid.org/0000-0002-0970-0439

Sang Seok Kim, https://orcid.org/0000-0001-9717-9710

\section{REFERENCES}

1. Wallace GF. Dermatologic causes of heel pain. Clin Podiatr Med Surg 2010;27:407-416.

2. Bernathova M, Bein E, Bendix N, Bodner G. Sonographic diagnosis of plantar vein thrombosis: report of 3 cases. J Ultrasound Med 2005;24:101-103.

3. Siegal DS, Wu JS, Brennan DD, Challies T, Hochman MG. Plantar vein thrombosis: a rare cause of plantar foot pain. Skeletal Radiol 2008;37:267-269.

4. Long A, Bura-Riviere A, Sapoval M. [Plantar venous thrombosis and anticardiolipin antibody syndrome. Case report]. J Mal Vasc 2004;29:39-40. French.

5. Cavezzi A. Isolated thrombosis of plantar veins. Case report. Minerva Cardioangiol 1999;47:309-313.

6. Legrand MS, Papon X, Leftheriotis G, Saumet JL. [Isolated plantar venous thrombosis. Report of a case]. J Mal Vasc 1997;22:364-365. French.

7. Lee KM, Park JH, Min KH, Kim EK. Epidermal cyst on the sole. Arch Plast Surg 2013;40:475-476.

8. Bae JM, Kang H, Kim HO, Park YM. Differential diagnosis of plantar wart from corn, callus and healed wart with the aid of dermoscopy. Br J Dermatol 2009;160:220-222. 
9. Thomas VD, Snavely NR, Lee KK, Swanson NA. Benign epithelial tumors, hamartomas, and hyperplasias. In: Goldsmith LA, Katz SI, Gilchrest BA, Paller AS, Leffell DJ, Wolff $K$, et al., editors. Fitzpatrick's dermatology in general medicine. 8th ed. New York: McGraw-Hill, 2012: 1319-1336.

10. Robbin MR, Murphey MD, Temple HT, Kransdorf MJ, Choi JJ. Imaging of musculoskeletal fibromatosis. Radiographics 2001;21:585-600.

11. Geiger C, Rademacher A, Chappell D, Sadeghi-Azandaryani
M, Heyn J. Plantar vein thrombosis due to busy night duty on intensive care unit. Clin Appl Thromb Hemost 2011;17: 232-234.

12. Fabro M, Fabro SR, Sales RS, Machado CA, de Araújo GL. Plantar vein thrombosis: a rare differential diagnosis in patients with plantar pain. Radiol Bras 2015;48:399-400.

13. Houdek MT, Warneke JA, Pollard CM, Lindgren EA, Taljanovic MS. Giant epidermal cyst of the gluteal region. Radiol Case Rep 2015;5:476. 